\title{
NITRIDATION OF GaAs (001)-2x4 SURFACE STUDIED BY AUGER-ELECTRON SPECTROSCOPY
}

\author{
Igor Aksenov $^{*}$, Yoshinobu Nakada ${ }^{* *}$ and Hajime Okumura ${ }^{* * *}$ \\ * Joint Research Center for Atom Technology (JRCAT) - Angstrom Technology Partnership, \\ ** Higashi 1-1-4, Tsukuba, Ibaraki 305, Japan, aksenov@jrcat.or.jp \\ Mitsubishi Materials Corporation, 1-297 Kitabukuro, Omiya, Saitama 330, Japan \\ Electrotechnical Laboratory, Umezono 1-1-4, Tsukuba, Ibaraki 305, Japan
}

Cite this article as: MRS Internet J.Nitride Semicond.Res.4S1, G3.4 (1999)

\begin{abstract}
Auger electron spectroscopy (AES) was used to investigate the processes taking place during the initial stages of nitridation of GaAs (001) surface. The analysis of the AES results combined with that of RHEED show that the processes taking place during nitridation greatly differ depending on the nitridation temperature. At low temperatures $\left(\leq 200^{\circ} \mathrm{C}\right)$ nitridation is hindered by kinetic restrictions on atomic migration, whereas at high temperatures $\left(\geq 500^{\circ} \mathrm{C}\right)$ the process of nitridation takes place simultaneously with the etching of the surface. However, for intermediate temperatures $\left(300^{\circ} \mathrm{C} \sim 400^{\circ} \mathrm{C}\right)$ the results indicate that a complete monolayer of $\mathrm{N}$ atoms may be formed on the substrate during the initial stage of nitridation. The post-nitridation annealing of the samples nitrided at the intermediate temperatures results in the formation of a crystalline GaN layer, the line shape of the AES signals from which is identical to that for a $\mathrm{GaN}$ reference sample.
\end{abstract}

\section{INTRODUCTION}

stigate the processes taking place during the initial stages of interaGaN compound is difficult to grow on the technologically well-established substrates, i.e. Si and GaAs, which is due to the significant lattice mismatch of $\mathrm{GaN}$ with those materials. One of the methods of overcoming that difficulty is to grow a thin "low-temperature" buffer layer on the top of GaAs, which is amorphous and which crystallizes upon thermal annealing, leading to relaxation of the lattice mismatch. The subsequent MBE growth of the GaN compound on the top of that buffer layer results in a good quality cubic phase GaN [1]. However, a deeper understanding of the mechanisms governing initial stages of nitridation and subsequent nitride growth is vital for improvement of device performance. The formation of Ga-N and As-N bonds on the GaAs (001) surface during nitridation has been verified by X-ray photoelectron spectroscopy (XPS) [2,3], the results showing chemical shifts of the core levels of $\mathrm{Ga}$, As and $\mathrm{N}$ caused by the nitridation process, with the formation of a disordered Ga-As-N phase in the subsurface region of the substrate. However, all XPS experiments have been carried out after nitridation by either transferring the nitrided sample from the "nitridation" chamber to the "XPS" chamber or by switching between nitridation and measurement cycles in the same chamber. It is important to note the inborn limitations of this experimental protocol since there is no guarantee that the surface conditions during measurements will be the same as those directly after the nitridation.

In the present study we used AES to invection of active nitrogen species with the GaAs (001)-2x4 surface at various surface temperatures in the range from room temperature (RT) to $600^{\circ} \mathrm{C}$. The measurements have been carried out simultaneously with the nitridation process, providing, thereby, an insight into the process of nitridation not hindered by the "postnitridation" relaxation effects.

\section{EXPERIMENT}

An experimental machine combining a differentially pumped electron beam column, a hemispherical electron energy analyzer for AES, as well as an RF plasma source for generation of active nitrogen species, has been constructed. Differential pumping of the column allows us to 
make AES measurements in the process of supplying active nitrogen to the surface, when the pressure in the chamber goes up to $10^{-5}$ Torr (base pressure is $2 \times 10^{-10}$ Torr). The surface studied was an MBE-regrown GaAs (001) surface exhibiting (2x4) surface reconstruction, and the nitridation rate was controlled by using a mass flow controller, through which a flow of $1 \mathrm{ccm} / \mathrm{s}$ of the $\mathrm{N}_{2}$ gas was introduced into the $\mathrm{N}$-source. The substrate temperature during nitridation $\left(\mathrm{T}_{\mathrm{s}}\right)$ was kept constant at various levels, i.e. at $\mathrm{T}_{\mathrm{s}}=\mathrm{RT}, 200^{\circ} \mathrm{C}, 300^{\circ} \mathrm{C}, 400^{\circ} \mathrm{C}, 500^{\circ} \mathrm{C}$ and $600^{\circ} \mathrm{C}$. To characterize the bonding and composition of the buffer layer growing during the nitridation process, the Auger signals of Ga-LMM, As-LMM and N-KLL transitions at about 1070, 1230 and $380 \mathrm{eV}$, respectively, have been used. No traces of impurities, such as oxygen and carbon, have been detected on the original surface and at the beginning of the nitridation processes, although some oxygen $(<2 \%)$ tends to appear after prolonged $(\sim 2 \mathrm{~h})$ exposures to nitrogen flux.

\section{RESULTS}

\section{Spectral Shape of the Auger Signals and the Core Level Shifts}

The detailed non-differentiated spectra of N, Ga and As Auger transitions for various Nexposures are shown in Fig. 1 for $\mathrm{T}_{\mathrm{s}}=300^{\circ} \mathrm{C}$. It can be seen that the signals exhibit, in addition to the dominant peak, a shoulder on the high kinetic energy (KE) side of the spectra. The experimental spectra can be deconvoluted into Voigt doublets (not shown), with the energy separation between the Voigt peaks constituting each doublet corresponding to the splitting of the LMM- and KLL-multiplets taking place due to the difference in the total relaxation energy of two core holes created during the Auger process. In addition, the As-signal during nitridation also exhibits a shoulder at the low-KE side of the spectra, this shoulder becoming more pronounced with decrease in the nitridation temperature. On the basis of the previous XPS results the above shoulder has been attributed to the surface AsN species, and the disappearance of this peak at higher temperatures is caused by desorption of the AsN species from the surface [2,3]. The Noriginated spectra during nitridation also exhibit a shoulder at the low-energy side of the spectra separated by $\sim 2.0 \mathrm{eV}$ from the main N-KLL peak (Fig. 1), which disappears at higher temperatures just like the low-KE side shoulder on the As-originated spectra. This shoulder has been assigned to the N-originated signal from As- $\mathrm{N}$ bonds in the AsN species, in accordance with XPS results [3].

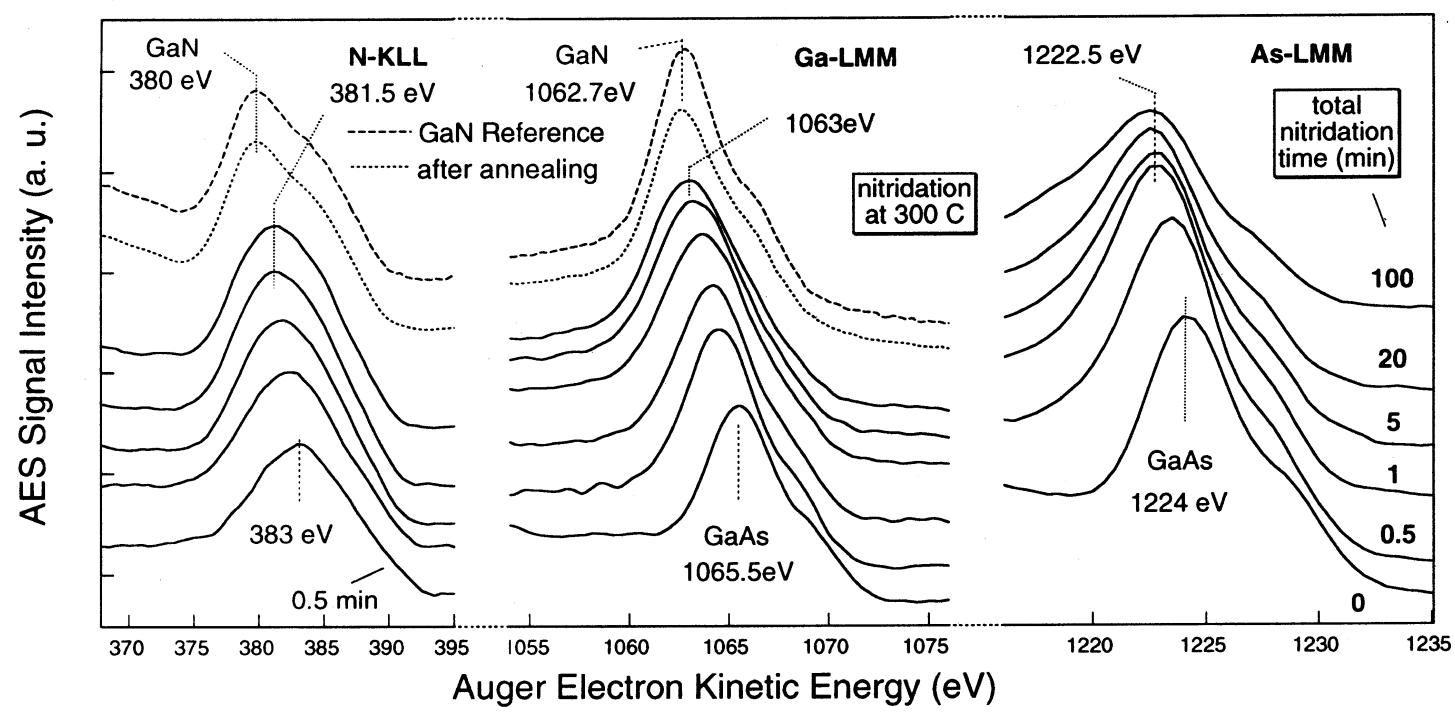

Fig. 1. Detailed Auger spectra from N-KLL, Ga-LMM and As-LMM transitions for various nitrogen exposures for the nitridation temperature $\mathrm{T}_{\mathrm{s}}=300^{\circ} \mathrm{C}$, together with that of $\mathrm{N}$ and Ga taken after subsequent annealing at $630^{\circ} \mathrm{C}$ for $5 \mathrm{~min}$ (dotted curves) and those for a thick $(\sim 1.5 \mu \mathrm{m}) \mathrm{MBE}$-grown GaN reference sample (dashed curves). 
It can be seen from Fig. 1 that the spectral positions of the peaks exhibit shifts to lower KE as the nitridation proceeds. It has been found that the magnitude of these shifts depends on the nitridation temperature. Dependencies of the apparent energy shift of the peak of Ga-signal on nitrogen exposure for various nitridation temperatures are shown in Fig. 2. It can be seen that the magnitude of the shift increases with increase in the nitridation temperature in the range $\mathrm{RT} \leq \mathrm{T}_{\mathrm{s}}$ $\leq 300^{\circ} \mathrm{C}$, levels off for $300^{\circ} \mathrm{C} \leq \mathrm{T}_{\mathrm{s}} \leq 400^{\circ} \mathrm{C}$, and starts to decrease for $\mathrm{T}_{\mathrm{s}} \geq 500^{\circ} \mathrm{C}$. Therefore, the

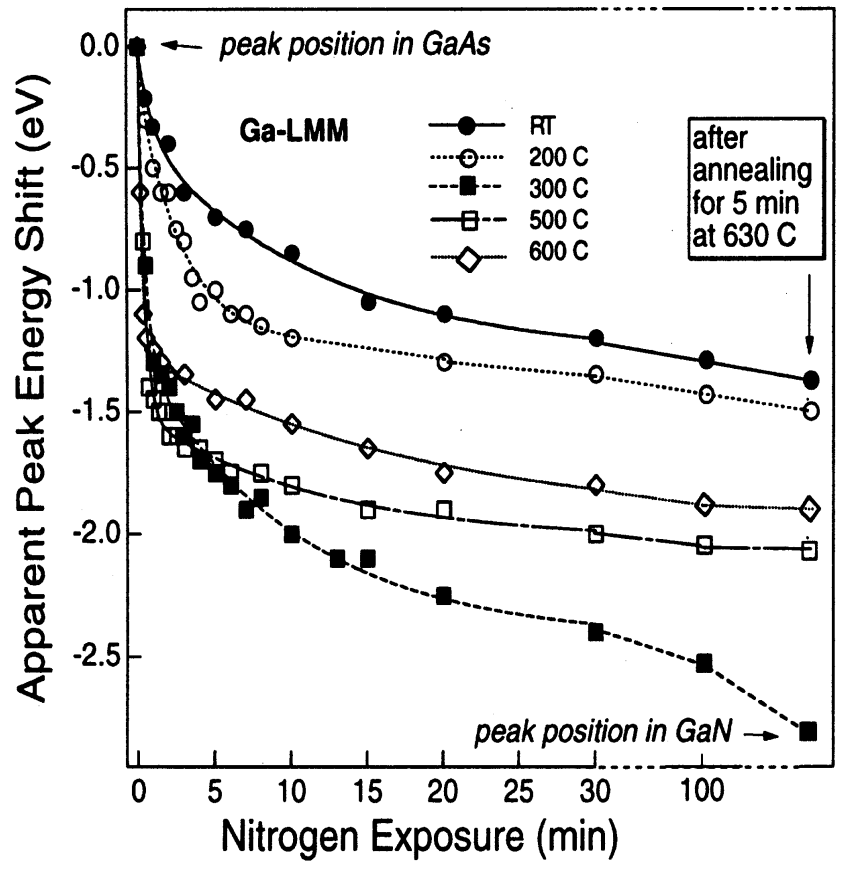

Fig. 2. Dependencies of the energy shifts of the peak of Ga-transition on $\mathrm{N}$-exposure for various substrate temperatures together with that after subsequent annealing at $630^{\circ} \mathrm{C}$ for $5 \mathrm{~min}$. The data for $400^{\circ} \mathrm{C}$ is similar to that for $300^{\circ} \mathrm{C}$ and is not shown. taken from the surface of a semiconductor. The first one is a chemical shift reflecting change in a chemical environment and the second is a shift of the Fermi level due to band bending. As it has been verified by XPS studies [2,3], nitridation of the GaAs (001) surface results in the formation of the disordered Ga-As-N compound as incoming $\mathrm{N}$ atoms substitute for the As atoms. The formation of the Ga-As-N phase leads to the broadening of the Auger signals and is partly responsible for the observed shifts in the spectra. Since the ionicity of the Ga-N bonds is higher than that of the Ga-As bonds due to an increased charge transfer from Ga to anion as arsenic is substituted by nitrogen, we can expect a shift of the core levels of Ga to higher binding energies (BE) and, therefore, a low-KE shift of the Ga-LMM Auger transition, which is in accordance with the experimental results. Since the low-KE shift of the spectra is mostly pronounced during the first 2 - 5 min of nitridation, we believe that the nitridation of the surface takes place mostly during this short time interval, after which it is much slowed down as $\mathrm{N}$ atoms begin to substitute As atoms in the back-bonds.

Comparing N-As bonds with the Ga-As ones we can see that the direction of charge transfer is reversed, the charge being transferred from As atom in the $\mathrm{N}$-As bonds. Therefore, the formation of the N-As bonds should result in a low-KE shift of the As-originated signal, which is observed experimentally, but only for the nitridation temperatures $T_{\mathrm{s}} \leq 400^{\circ} \mathrm{C}$. At higher temperatures the As-originated signal, while shifting a little towards low-KE during the first 2 min of nitridation, exhibits a small shift towards high-KE as the nitridation proceeds. Therefore, we believe that the shift of the As-signal is determined by an interplay of two (or several) effects. 
Careful analysis of the above results, together with those for the $\mathrm{N}$-originated signal, indicate that, whereas nitridation at low temperatures $\left(\mathrm{T}_{\mathrm{s}}<300^{\circ} \mathrm{C}\right)$ results only in the chemical shift of the Auger signals under consideration, that for the temperatures $\mathrm{T}_{\mathrm{s}} \geq 300^{\circ} \mathrm{C}$ leads to both the chemical shift and a shift caused by the band bending effect. It remains unclear why the band bending effect is the most pronounced at the temperatures in the range $300^{\circ} \mathrm{C}-400^{\circ} \mathrm{C}$. One possible explanation may be that a uniform layer of $\mathrm{GaN}$ (possibly creating a heterojunction with GaAs) is formed on the surface at these nitridation temperatures, which, however, is not formed upon nitridation at lower or higher temperatures due to kinetic restrictions on atomic migration (with the resulting island-like structures on the surface) or roughening of the surface due to etching effects for lower and higher temperatures, respectively.

\section{Dependencies of the Auger Intensities on Nitrogen Exposure}

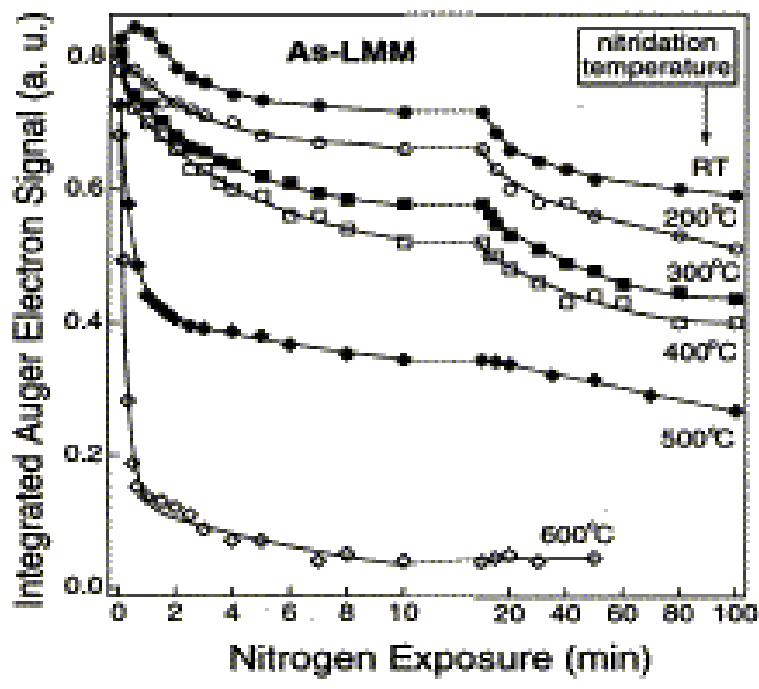

Fig. 3. Dependencies of As-signal intensities on the $\mathrm{N}$-exposure for various temperatures.

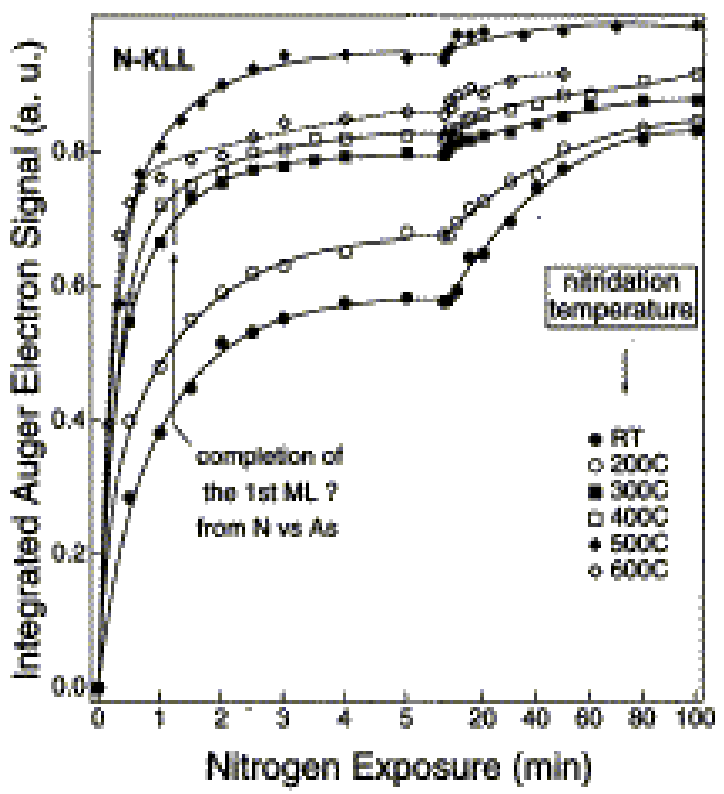

Fig. 4. Dependencies of N-signal intensities on the $\mathrm{N}$-exposure for various temperatures.
Figs. 3, 4 show the dependencies of the integrated intensities $I(t)$ for the As- and Noriginated Auger signals on the nitrogen exposure time $t$ during nitridation. Since the spectra of all three elements exhibit a change in their spectral shape as the nitridation proceeds, we could not use the peak-to-peak values of the differentiated Auger signals to determine intensities of the signals. Instead, the areas of non-differentiated signals have been used for quantitative evaluation of the nitridation process, the areas having been determined by a trapezoidal integration with the cosine background correction. The data for the intensities the As- and $\mathrm{N}$ - originated signals has been normalized to that of the Ga-originated signal prior to the nitrogen exposure, the intensity of which at any nitridation temperature was taken as 1.0.

It can be seen from Figs. 3, 4 that, as the nitridation proceeds, the intensity of the Assignal decreases,

whereas that of the N-signal increases. At the same time, the intensity of Ga-signal does not exhibit any drastic changes (not shown). It can also be seen that the intensities of the As- and N-originated signals exhibit the most drastic changes during the first several min (or even seconds, depending on the temperature) of nitridation, after which the signals level off at the levels strongly dependent upon the nitridation temperature. The magnitude of the changes in the intensities of the $\mathrm{N}$ - and As-originated signals greatly exceeds that for the Ga-originated signal, which is consistent with the substitutional (anion exchange) nature of the nitridation process, when $\mathrm{N}$ atoms substitute for those of As, whereas $\mathrm{Ga}$ atoms mostly retain their positions in the subsurface region.

An increase in the nitridation temperature results in a drastic decrease in the intensity of the As-signal due to the increase in the desorption rate of As and in a drastic increase in the 
intensity of the N-signal. However, it should be noted that, although the rate of an increase in the intensity of the N-signal (related to the sticking coefficient) for the highest nitridation

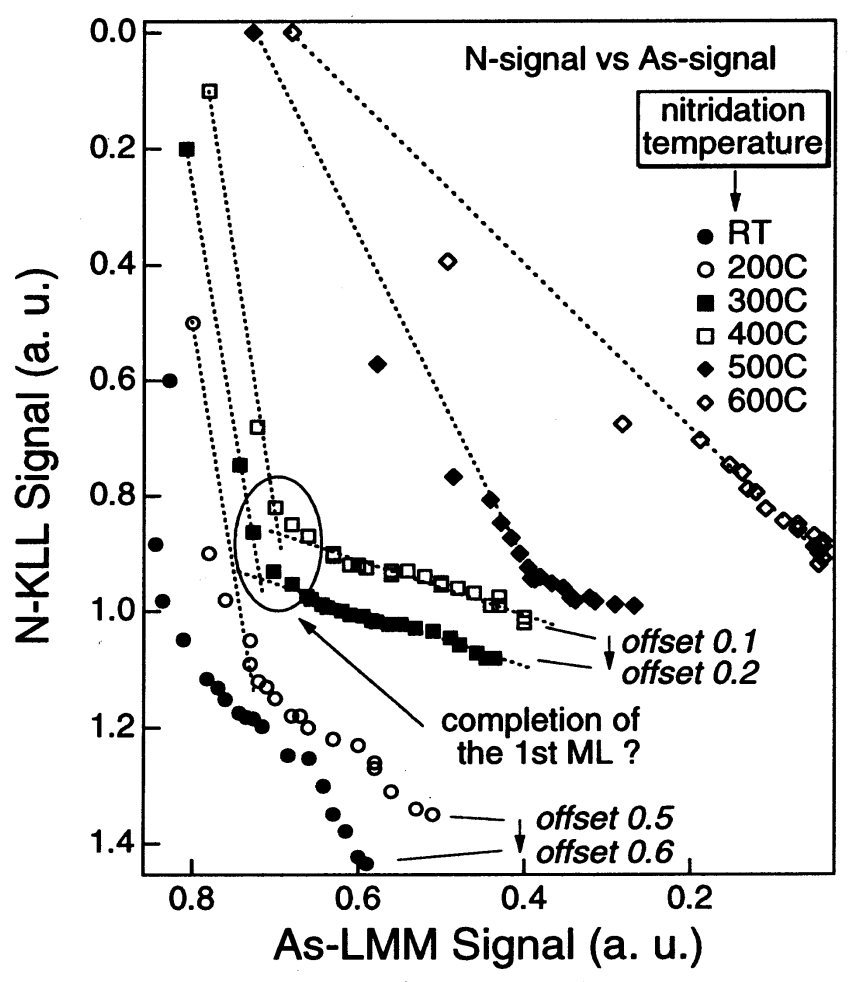

Fig. 5. Intensities of the $\mathrm{N}$-signal as a function of that for the As-signal for various temperatures. Ellipse shows the position of breaks on the curves which probably arise due to the completion of the first monolayer. Notice the offsets of the curves. temperature studied $\left(600^{\circ} \mathrm{C}\right)$ during the initial $40 \mathrm{sec}$ of nitridation exceeds that for $500^{\circ} \mathrm{C}$, further nitridation results in the leveling off the signal for $\mathrm{T}_{\mathrm{S}}=600^{\circ} \mathrm{C}$ at the level lower than that for $\mathrm{T}_{\mathrm{s}}=500^{\circ} \mathrm{C}$. The last phenomenon can be explained by taking into account an increased desorption rate of the nitrogen species from the nitrided surface at $\mathrm{T}_{\mathrm{s}}=600^{\circ} \mathrm{C}$, which is accordance with the results of Makimoto et al . [6].

The dependencies of intensities of both As- and N-signals (not shown) are exponential-like smooth curves exhibiting no apparent breaks. However, plots of intensities of the N-originated signal (adsorbate) against that of the Asoriginated one (substrate), shown in Fig. 5, exhibit pronounced breaks, but only for the data taken for nitridation temperatures $\mathrm{T}_{\mathrm{s}}=$ $300^{\circ} \mathrm{C}$ and $400^{\circ} \mathrm{C}$ (see ellipse in Fig. 5). A break in an adsorbate signal $v s$ substrate signal dependence usually occurs at the completion of a monolayer (ML) [5]. Therefore, the breaks on the curves shown in Fig. 5 are believed to correspond to the completion of the first ML of $\mathrm{N}$ atoms.

The position of the break-points in Fig. 5 corresponds to the nitridation time of about $1.5 \mathrm{~min}$ and is shown as a vertical line on the $I_{N}(t)$ dependencies in Fig. 4. It can be seen from the examination of this figure that no apparent breaks are discernible in the $I_{N}(t)$ dependencies after 1.5 min of nitridation, which seems to contradict the results shown in Fig. 5 . The above discrepancy, however, can be explained by taking into account possible changes of sticking probability of the active nitrogen species [7]. Indeed, whereas gradual changes in the value of the sticking probability result in the smoothing of the $I_{A s}(t)$ and $I_{N}(t)$ dependencies, it will not affect the plot of $I_{N} v s \quad I_{A s}$ since the time-variable is eliminated in this plot.

\section{RHEED Results}

Nitridation results in drastic changes of the initial (2x4) RHEED pattern, these changes depending on the nitridation temperature. At low temperatures $\mathrm{T}_{\mathrm{s}} \leq 200^{\circ} \mathrm{C}$, once the nitrogen plasma is struck the GaAs reconstruction features fade out, the intensity of the diffracted beam being transferred to the background (not shown). The $(2 \times 4)$ reconstruction of the initial surface is destroyed after $1.5 \mathrm{~min}$ of nitridation with an appearance of (1x1) pattern from the unreconstructed surface, which, in its turn, disappears after 3 min of nitridation, giving rise to no pattern except for a smooth background, indicating that a disordered GaAsN layer is formed in the subsurface region. Thermal annealing of the samples at $630^{\circ} \mathrm{C}$ results in an appearance of an arc pattern, indicating an appearance of preferred orientations in the disordered layer. The crystallization, however, is not complete since the diffraction features retain their arc-like character even after prolonged annealing.

The situation is different for $\mathrm{T}_{\mathrm{S}}$ in the range $300^{\circ} \mathrm{C}-400^{\circ} \mathrm{C}$. Similar to the above case, nitridation during the first several min results in the formation of the disordered layer. However, after approximately 10 min of nitridation that disordered layer begins to re-crystallize, which is 
evidenced by an appearance of diffuse spots in the RHEED pattern corresponding to the lattice spacing of GaN (4.5 $\AA$ ). The thermal annealing then results in an appearance of the sharp spotty RHEED pattern characteristic of a mixture of cubic and hexagonal GaN phases, the cubic phase being dominant [1].

An increase in the nitridation temperature up to $\mathrm{T}_{\mathrm{s}} \geq 500^{\circ} \mathrm{C}$ results in a different progression. Upon striking the nitrogen plasma the $(2 \times 4)$ reconstruction quickly (after $\sim 20 \mathrm{sec}$ ) changes to a sharp streaked $(3 \times 3)$ pattern. However, the surface retains the $(3 \times 3)$ reconstruction for only 10 sec, after which the $(3 \times 3)$ features fade giving rise to the $(1 \times 1)$ pattern from the unreconstructed surface. The $(1 \times 1)$ pattern, in its turn, also fades as the nitridation proceeds, but after further 2 min of nitridation the GaN-originated spots, superposed on the blurred (1x1) streaked pattern from GaAs, become discernible in the pattern. As we proceed with nitridation, the intensity of the GaN-originated spots increases, whereas that of the GaAs-originated streaks decreases, the streaks becoming indiscernible after about $5 \mathrm{~min}$ from the beginning of nitridation. At the same time, however, a blurred spots corresponding to the (004)- and (115)-diffraction features from the GaAs phase appear in the pattern, the intensity of this spots increasing as we proceed with nitridation. Diagonal lines between the diffraction spots also appear in the pattern, which is due to the formation of the (111) facets caused, in its turn, by the etching of the substrate by impinging nitrogen species [1]. Annealing of the samples at $650-700^{\circ} \mathrm{C}$ results in a disappearance of the GaAs-originated spots in the pattern. Therefore, we believe that at high nitridation temperatures the GaAs islands are formed on the top of the nitrided GaN layer due to out-diffusion of arsenic, substituted by nitrogen, from the GaAs substrate. These islands desorb from the surface at the temperatures higher than $650^{\circ} \mathrm{C}$ due to the desorption of arsenic.

\section{CONCLUSIONS}

In conclusion, the results presented above show that the processes taking place during nitridation of the GaAs (001) surface greatly differ depending on the nitridation (surface) temperature. On the one hand, at low temperatures $\left(\mathrm{T}_{\mathrm{s}} \leq 200^{\circ} \mathrm{C}\right)$ nitridation is hindered by kinetic restrictions on the atomic migration, and we believe that nitridation occurs through the formation of islands or "simultaneous multilayers" which do not cover the underlying surface completely. Disordered GaAsN phase is formed in the subsurface region, and that phase can not be completely re-crystallized even after prolonged annealing.

On the other hand, at high temperatures $\left(\mathrm{T}_{\mathrm{s}} \geq 500^{\circ} \mathrm{C}\right)$ the process of nitridation takes place simultaneously with the etching of the surface (at different portions of the surface) due to decomposition of the substrate under an impinging N-flux, leading to the growth of the (111) facets and resulting in the rough interface between the $\mathrm{GaN}$ and $\mathrm{GaAs}$ phases. It seems that the $\mathrm{N}$ atoms agglomerate to the nitrided portions of the surface to form $\mathrm{GaN}$ islands, whereas the surface portions which are not nitrided initially are etched by exposing the (111) facets.

However, for intermediate nitridation temperatures $\left(300^{\circ} \mathrm{C} \leq \mathrm{T}_{\mathrm{s}} \leq 400^{\circ} \mathrm{C}\right)$ it was found that the disordered GaAsN phase, formed at the initial stage of nitridation, partly crystallizes even without annealing. Moreover, AES results indicate that one monolayer of $\mathrm{N}$ atoms (partly mixed with arsenic) may be formed on the substrate during the initial stage of nitridation. The formation of the complete ML of GaN results in the band bending, which leads to the most pronounced low kinetic energy shifts of the Auger signals for the nitridation temperatures in this range. The postnitridation annealing of the samples nitrided at the intermediate temperatures results in the formation of a crystalline GaN layer, the line shape of the AES signals from which is identical to that for a thick $\mathrm{GaN}$ reference sample.

\section{REFERENCES}

1. H. Okumura, K. Ohta, G. Feuillet, K. Balakrishnan, S. Chichibu, H. Hamaguchi, P. Hacke, and S. Yoshida, J. Cryst. Growth 178, 113 (1997).

2. A. Masuda, Y. Yonezawa, A. Morimoto, and T. Shimizu, Jpn. J. Appl. Phys. 34, 1075 (1995).

3. X.-Y. Zhu, M. Wolf, T. Huett, and J. M. White, J. Chem. Phys. 97, 5856 (1992).

4. I. Aksenov, Y. Nakada, and H. Okumura, Jpn. J. Appl. Phys. 37, L972 (1998).

5. C. Argile and G. E. Rhead, Surf. Sci. Rep. 10, 277 (1989).

6. T. Makimoto and N. Kobayashi: Appl. Surface Sci. 100/101, 403 (1996).

7. I. Aksenov, Y. Nakada, and H. Okumura, J. Appl. Phys. 84, 3159 (1998). 